\title{
CONTRIBUIÇÕES DA CULTURA AFRO-INDIGENA PARA A MATEMÁTICA
}

\section{CONTRIBUTIONS OF AFRO-INDIGENOUS CULTURE TO MATHEMATICS}

\author{
Fransuelton Gomes Anastácioํㅜ ${ }^{1}$ Júnio Moreira de Alencar²; Luciana Maria de Sousa \\ Macêdo ${ }^{3}$
}

\begin{abstract}
RESUMO
O objetivo deste estudo foi relatar uma experiência de ensino e aprendizagem a distância via internet durante a Primeira Semana do Colóquio Temático do Ensino Fundamental e Médio Amália Xavier, na cidade de Juazeiro do Norte, Ceará, Brasil. Inicialmente, os professores demonstraram a relação entre a cultura afro-indígena e a matemática e suas contribuições para a construção do conhecimento lógico-matemático. Para atingir estes objetivos, foi apresentada uma breve explicação sobre dois jogos africanos: Mancala e Yoté, e suas regras e estratégias. Esses jogos eram muito comuns no cotidiano dos negros e, historicamente, relacionavam-se com o trabalho agrícola deles. As explicações sobre os jogos africanos foram feitas utilizando algum tipo deles, como a versão online e outras versões com materiais de baixo custo. Esta abordagem foi útil para motivar o desenvolvimento interativo do raciocínio lógico dos alunos. Durante as apresentações, foi possível refletir sobre as diversas contribuições dos indígenas e afrodescendentes não só na história brasileira, mas também no desenvolvimento científicomatemático. De acordo com nossa observação, alunos, pais, professores e todos os participantes apoiaram e foram positivos em relação ao uso dos jogos Mancala e Yoté na aprendizagem da matemática. Uma das reflexões mais interessantes sobre o processo ensino-aprendizagem da matemática por meio desses jogos foi que essa ciência se torna relevante e ainda mais bonita. Além disso, aprender matemática pode ser divertido com a influência da cultura dos povos afroindígenas e sua alegria e criatividade. Diante disso, os professores se sentiram motivados a
\end{abstract}

${ }^{1}$ Graduado em Licenciatura plena de Matemática (IFCE - Campus Juazeiro do Norte). Discente do Curso de Especialização lato sensu em Ensino de Matemática com ênfase na Formação de Professores da Educação Básica (IFCE - Campus Juazeiro do Norte), Juazeiro do Norte, Ceará, Brasil. Endereço para correspondência: Rua Magistoso Pinto da Fonseca 210, Casa, Frei Damião, Juazeiro do Norte, Ceará, Brasil, CEP: 63043-255. E-mail: fransuelton.junior@gmail.com.

iD ORCID iD: https://orcid.org/0000-0002-2949-8696.

${ }^{2}$ Mestre em Matemática Pura pela Universidade Federal do Ceará (UFC) e Doutor em Engenharia Agrícola pela Universidade Federal do Ceará (UFC). Professor de Ensino Básico Técnico e Tecnológico do departamento do Curso de Licenciatura plena de Matemática do Instituto Federal de Educação, Ciência e Tecnologia do Ceará (IFCE), Juazeiro do Norte, Ceará, Brasil. Juazeiro do Norte, Ceará, Brasil. Endereço para correspondência: Av. Plácido Aderaldo Castelo, 1646, Campus Juazeiro do Norte-CE, Planalto, Juazeiro do Norte, Ceará, Brasil, CEP: 63047-040. E-mail: juniomoreira@ ifce.edu.br.

(DORCID iD: https://orcid.org/0000-0001-7903-207X .

${ }^{3}$ Mestre em Ensino de Ciências e Educação Matemática pela Universidade Estadual da Paraíba (UEPB). Professora do Curso de Licenciatura em Matemática da Universidade Regional do Cariri (URCA), Pertencente ao grupo de estudo de Educação Matemática, Juazeiro do Norte, Ceará, Brasil. Endereço para correspondência: Av. Leão Sampaio, 107, Lagoa Seca, Juazeiro do Norte, Ceará, Brasil, CEP: 63041-145. E-mail: luciana.macedo@urca.br

(iD) ORCID iD: https://orcid.org/0000-0003-4415-934x. 
investigar mais o uso potencial do ensino da matemática por meio dos jogos Mancala e Yoté e aprender mais sobre a reinvenção do negro para transformar o impossível e o restrito, em algo possível e para o desfrute de todos.

Palavras-chave: Afro-indígena; Cultura; Conhecimento; Jogo; Lógica.

\begin{abstract}
The aim of this study was to report an experience of distance teaching and learning via internet during the First Thematic Colloquium Week of the Amália Xavier Elementary and High School, in the city of Juazeiro do Norte, Ceará, Brazil. Initially, teachers demonstrated the relationship between Afro-indigenous culture and mathematics and their contributions to the construction of logical-mathematical knowledge. In order to achieve these goals, a short explanation about two African games: Mancala and Yoté, and their rules and strategies was presented. Those games were very common in daily life of black people and hystorically, they had related with the agricultural work of them. The explanation about the African games were performed by using some type of them, such as an online version and other versions with low-cost materials. This approach was useful in order to motivate the interactive development of students' logical reasoning. During the presentations, it was possible to reflect on various contributions of indigenous and Afro-descedant not only in brazilian history but also in in scientific-mathematical development. According to our observation, students, parents, teachers and all participants were supportive and positive towards the use of Mancala and Yoté games in learning mathematics. One of the most interesting reflection on the teaching-learning process of mathematics through these games was that this science becomes relevant and even more beautiful. In addition, learning mathematics can be fun with the influence of Afro-indigenous people culture and their joy and creativity. In view of that, teachers felt motivated to investigate more the potential use of teaching mathematics through Mancala and Yoté games and learn more about the reinvention of black people to transform the impossible and restricted, in something possible and for everyone to enjoy.
\end{abstract}

Keywords: Afro-indigenous; Culture; Knowledge; Game; Logic. 


\section{Introdução}

A I Semana de Colóquios Temáticos da Escola de Ensino Fundamental e Médio Amália Xavier, do tipo regular, localizada na cidade de Juazeiro do Norte-CE, realizada entre os dias 09 a 13 de novembro de 2020, foi construída na proposta de incentivar uma melhor participação dos alunos no ensino remoto (Figura 1). Na sua programação, o evento temático tratou de aspectos históricos e culturais dos índios e negros e suas contribuições na obtenção dos fármacos que usamos nos chás e na construção e desenvolvimento de conceitos lógico-matemáticos.

Um momento bem significativo e atípico para todos se reencontrarem virtualmente, uma vez que se faz necessário o isolamento social devido ao cenário mundial causado pela pandemia da covid-19.

Figuras 1 - Encarte do Seminário com a programação.

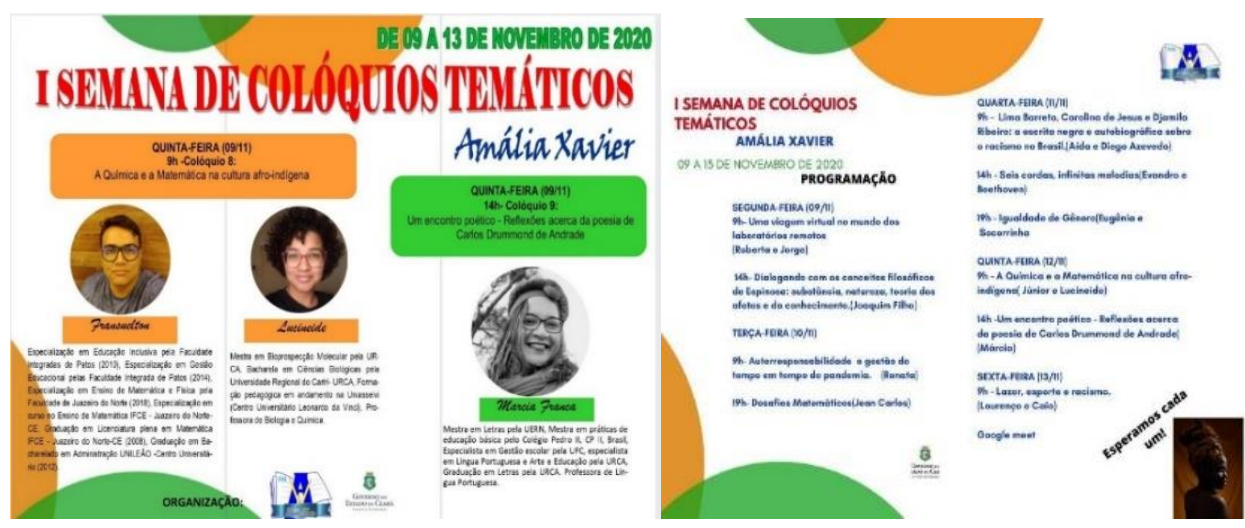

Fonte: Arquivo dos autores.

Uma atividade realizada no dia 12 de novembro, demonstrou, por meio da plataforma digital Google Meet, onde se reuniram todos os membros que fazem parte da escola - pais, alunos, professores, funcionários, ex-alunos e os membros da gestão -, o interesse por conhecer a relação entre a Matemática e a cultura Afro-indígena.

O presente trabalho tem o objetivo de relatar uma experiência de se trabalhar com uma temática interdisciplinar que possibilita o ensino interativo da Matemática com a História e Cultura africana e afro-brasileira, a partir dos jogos e outras manifestações culturais africana, muito presente em várias áreas do conhecimento, mas por vezes pouca divulgada na área de ciências da natureza e exatas.

\section{A contribuição Afro-indígena}


De acordo com Mota (2020, p.3), um dos objetivos dos Parâmetros Curriculares Nacionais (PCN's), para o Ensino Fundamental é

Conhecer e valorizar a pluralidade do patrimônio sociocultural brasileiro, bem como aspectos socioculturais de outros povos e nações, posicionando-se contra qualquer discriminação baseada em diferenças culturais, de classe social, de crenças, de sexo, de etnia ou outras características individuais e sociais. (BRASIL, 1997, p. 6).

O que está em consonância com as diretrizes curriculares nacionais quando sanciona a Lei de $\mathrm{n}^{\circ}$. 10.639/2003 para que haja um maior respeito aos aspectos socioculturais no contexto escolar, bem como promovera reflexão e ações de combate a todo e qualquer tipo de discriminação (BRASIL, 2003).

Observando a construção da história do nosso país e dos seus primeiros habitantes, percebe-se a presença de uma grande diversidade cultural impregnada em nosso jeito de falar, vestir, se alimentar e porque não pensar.

[...] um conjunto de hábitos, comportamentos, valores morais, crenças e símbolos, dentre outros aspectos mais gerais, como forma de organização social, política e econômica que caracterizam uma sociedade. Além disso, os processos históricos são em grande parte responsáveis pelas diferenças culturais. (RIBEIRO, 2019, p. 1).

Na produção do açúcar em solo brasileiro, os negros eram considerados como a mão de obra mais qualificada, pois já haviam sido escravizados na atividade açucareira na Península Ibérica. E nesse contexto, se deu a transmissão de saberes etnomatemáticos, pois agregavam elementos de sua cultura à matemática através dos jogos de raciocínio lógico construídos por meio de elementos de refugo das plantações como o Mancala e o Yoté. De acordo com Freyre (2001) é possível identificar a influência africana na figura das "mães-pretas" ou "mulatas de fino trato" transferiam para os "bem-nascidos da época" as cantigas, brincadeiras, estórias e contos, culinária, indumentária, danças e o culto religioso.

O Mancala (Figura 2) é um jogo simples, prático e intuitivo que traz consigo conceitos e operações da lógico-matemática, pode ser jogado a partir de dois jogadores, muito usado como um dos principais artifícios para brincar e interagir entre os povos quilombolas. Possuem um conjunto de espaços destinados a alocações de suas pedras 
para contabilizar as pontuações dos jogadores, lembrando a cova do milho e do feijão, tão presentes em seu trabalho.

Figuras 2 - Partidas de Mankala.

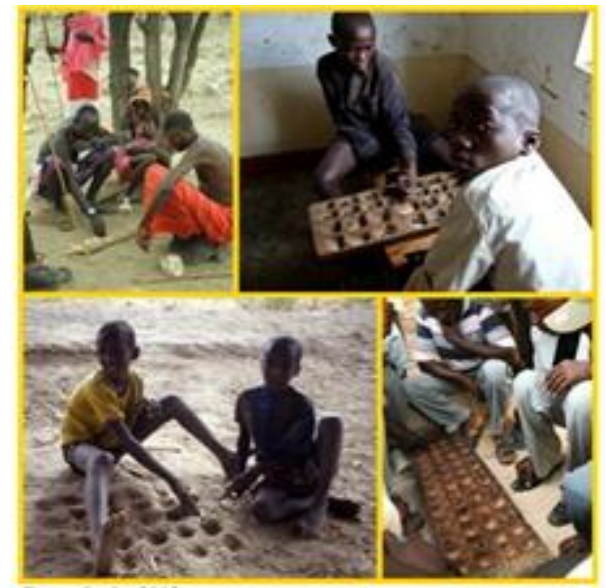

Fonte: Socio, 2013.

Este jogo possui uma estrutura que pode ser adaptada, a materiais recicláveis ou mesmo a um tabuleiro feito com tronco de árvores, entre outros (Figuras 3 a 6).

Figuras 3 - Foto de um tabuleiro de Mankala Colhe Três.

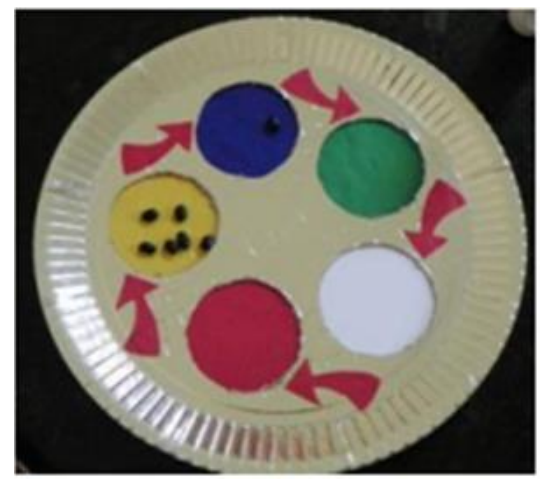

Fonte: Andrade et al, 2011.

Figuras 4 - Representação do Mancala com copos descartáveis. 


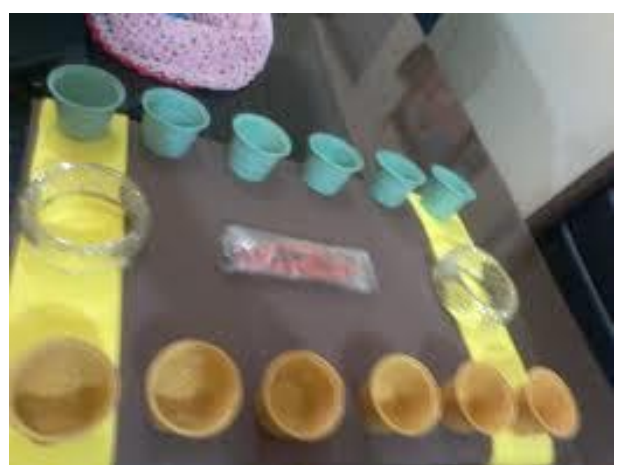

Fonte: https://www.youtube.com/watch?v=I8xROnVNCjY.

Figuras 5 e 6: Tipos de tabuleiro do Mancala.

Tabuleiro adaptado com potes de argila e folhas.

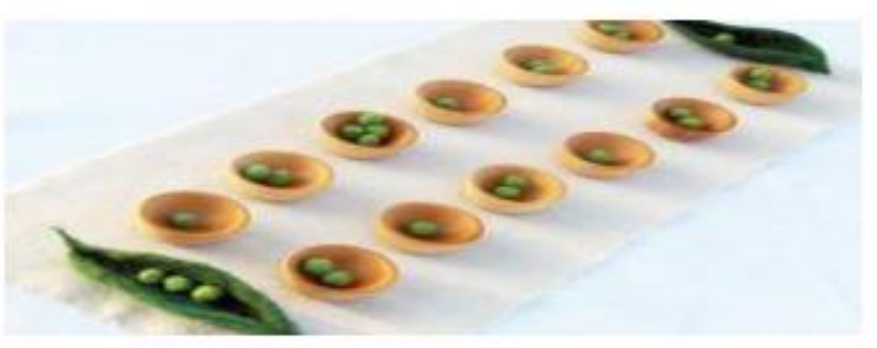

Tabuleiro adaptado com caixa de ovos e vasilhas de vidro.

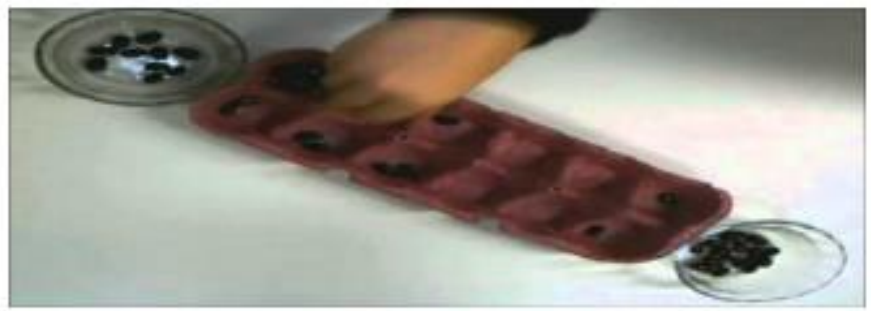

Fonte: Barreto, 2016.

O Yoté é um jogo de estratégia criado pelos povos africanos. Ele pode ser praticado por dois ou mais jogadores/as (como a dama e o xadrez), e é encontrado em vários países da África Ocidental, tais como Senegal, Gâmbia e Guiné. Utiliza um tabuleiro semelhante ao do jogo de dama ou xadrez (Figuras 7 e 8) com 64 casas e 24 peças ou no seu modelo usual de 30 casas e 24 peças distribuídas de acordo com a estratégia de cada jogador.

Figura 7 - Representação do Yoté. 


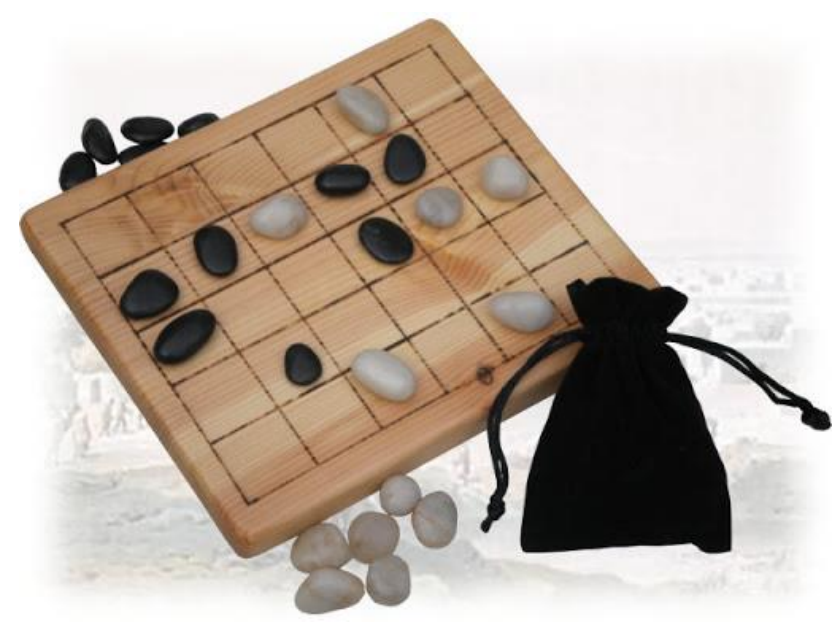

Fonte: http://www.cyningstan.com/game/342/yot

Figuras 8 - Representação do Yoté.

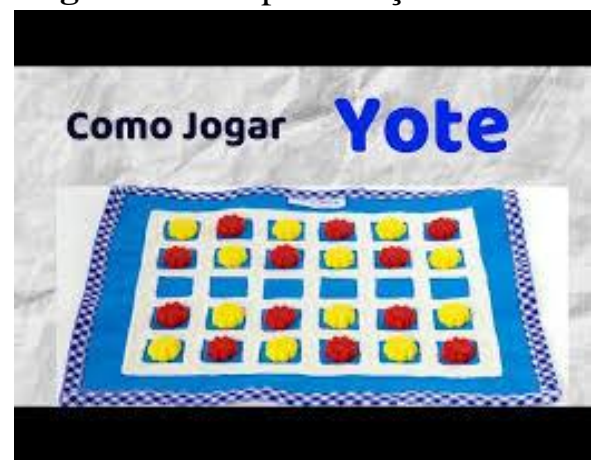

Fonte: https://www.youtube.com/watch?v=I8xROnVNCjY.

A pedras desse jogo podem ser substituídas por sementes, pedrinhas ou qualquer outro artifício e é facilmente adaptável para ser jogado no chão. Sua prática está associada historicamente a superação e adaptação das situações negativas dos negros e a busca por melhor qualidade. (Mais detalhes ver https://bodogemu.com/pt/games/yote).

\section{Jogos africanos que despertam o raciocínio}

$\mathrm{Na}$ atividade desenvolvida no evento temático foi apresentado os jogos Mancala e Yoté, de origem afro-indígena, como uma forma lúdica de entender as contribuições afrodescendente à nossa cultura, economia e o desenvolvimento cientifico-matemático. Em particular, foi relacionada a cultura negra com a abordagem Etnomatemática (D’AMBRÓSIO, 2005). 
Foram apresentados slides com a contextualização histórica e etnomatemática dos dois jogos afro-indígenas semelhantes ao da Figura 9.

Figura 9 - Jogo Mancala.

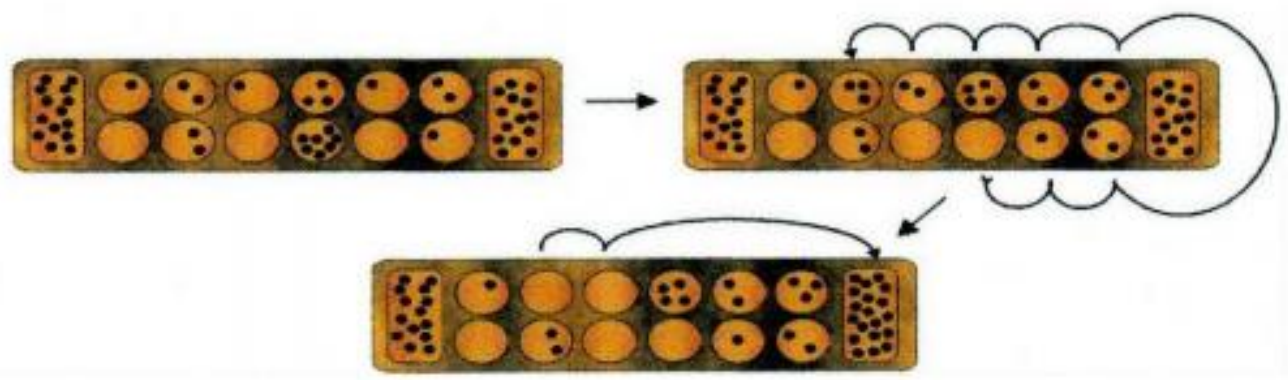

Fonte: Barreto, 2016.

Os dois jogos, foram ilustradas as regras e as maneiras de jogar do Mancala e do Yoté conforme pode ser encontrado em Fogaholi (2016) e no sítio https://bodogemu.com/pt/games/yote. Também, foram apresentadas as formas de construção dos jogos por meio de material de baixo custo ou mesmo reciclável e versões virtuais para computadores e celulares.

Apresentou-se as diversas variações dos jogos Mancala de acordo com a origem geográfica e foram abordados os contextos místico-religioso discutidos por Macedo (2000). No final da apresentação, abriu-se um espaço para discussão entre alunos, professores, gestores, funcionários, pais de alunos, alunos e ex-alunos sobre a significação da história com a matemática, do próprio jogo em si com a cultura negra e sua transmissão para as gerações futuras. Também foram relatadas experiências de vida e conexão com o tema do encontro e claro construção de novos saberes, tais como: uso da lógica num jogo de operações, transdisciplinaridade com o estudo da história e geografia, e promoção da coletividade e compartilhamento numa brincadeira sadia.

Para apresentar os jogos de origem africana e contar um pouco da presença histórica dos pretos em nosso território, foram realizadas pesquisas em livros, artigos científicos e sites que contavam um pouco desta temática, caracterizando uma pesquisa bibliográfica e exploratória. Segundo Gil (2002, p. 41), estas pesquisas têm como objetivo proporcionar maior familiaridade com o problema, com vistas a torná-lo mais explícito ou a constituir hipóteses. 


\section{Metodologia}

O trabalho em tela foi desenvolvido durante a I Semana de Colóquios Temáticos da Escola de Ensino fundamental e Médio Amália Xavier, localizado na cidade de Juazeiro do Norte - Ceará, no período de 09 a 13 de novembro de 2020. O momento em que realizamos a apresentação do tema proposto, exposição dos jogos, e abrimos um espaço para discussão acerca da temática Matemática e a Cultura Afro-indígena, em meio remoto, tendo a duração de uma hora e trinta minutos.

A clientela atendida por esta instituição de ensino é bem diversificada, contendo indivíduos da zona urbana e rural, devido ser umas das Escolas de Ensino Médio do município de Juazeiro do Norte-CE que oferece a modalidade regular, onde o discente estuda apenas em um dos horários dos três horários, dando a oportunidade de os mesmos estudarem em determinado turno de escolha e trabalharem em outro.

Devido ao isolamento social, o trabalho foi realizado por meio do Google Meet, contando com um total de 100 pessoas, sendo estás gestores, professores, pais, alunos e funcionários da escola.

Iniciamos o nosso trabalho demonstrando a relação entre a cultura afro-indígena e a Matemática. Buscamos durante o evento apresentar as contribuições que a cultura afro-indígena traz para a construção do conhecimento lógico matemático.

Para tanto, exploramos os jogos Mancala e o Yoté, apresentando suas regras, objetivos e as diferentes representações que venham possibilitar uma discussão sobre uma forma de fazer Matemática dentro do cotidiano dos povos negros em suas poucas horas lazer.

\section{Resultados}

O desenvolvimento da atividade permitiu contemplar as indicações dos Parâmetros Curriculares Nacionais (BRASIL, 1997) que defendem a importância de se trabalhar a Matemática para a construção da cidadania, salientando a necessidade da formação para o mundo do trabalho, bem como das relações sociais e da cultura na sociedade brasileira, destacando que "a pluralidade de etnias existentes no Brasil, que dá origem a diferentes modos de vida, valores, crenças e conhecimentos, apresenta-se para a educação matemática como um desafio interessante" (BRASIL, 1997, p.21). 
Nos jogos africanos, extraiu-se uma lição importante que difere de outros jogos de origem europeia, onde há construção de que, o ganhador é o que consegue chegar "sozinho" ao pódio, mas sim, o ganhador é aquele que divide e compartilha com os demais as 'sementes' ou 'pedras' do jogo. O coletivo é valorizado e não apenas o individualismo do prêmio ao ganhador. O conceito de divisão/ compartilhamento é ensinada desde cedo às crianças e jovens, e o que torna divertido o jogo é a sua capacidade de disputa entre os jogadores de quem será o primeiro a compartilhar.

Foi possível trabalhar o conceito da palavra ubuntu que segundo Moraes (2019, p. 32) é uma palavra proveniente da junção entre $u b u$ que remete a ideia de Ser, sendo a força vital, e $n t u$, aspectos particulares de uma existência, promovendo dois caráteres de existência, ao mesmo tempo uma existência que é anterior a ela mesma e uma existência que é posterior.

Notou-se que o intercâmbio cultural entre os negros africanos, indígenas e portugueses foram intensos, notadamente na língua, costumes, modos, comidas, forma de pensar e práticas religiosas. Chamando a atenção de que as trocas culturais e os contatos entre povos de origem muito diversa é algo que, então, fazia parte do dia a dia colonial, desde a chegada dos portugueses (PAIVA, 2001, p. 185).

Com esta atividade e através das discussões e momentos de escuta, foi possível refletir sobre a relação dos negros com a matemática e construir novos saberes etnomatemáticos como o uso de sementes como peças de um jogo tão simples que pode ser jogado fazendo ‘covas’ no chão. Os participantes puderam desmistificar a Matemática como uma ciência distante das relações humanas. E nesse contexto, promoveu o ensino de Matemática valorizando os conhecimentos prévios do educando e as diferentes formas de saberes dos grupos culturais que está de acordo com (Mota et al, 2020).

A presente experiência mostrou-se como uma experiência grandiosa e significativa para os envolvidos. Foi um momento de muita troca e compartilhamento de ideias, que motivaram a se fazer conhecer a temática para mais estudantes e estudioso da matemática, fazendo jus a Lei Federal n. 10.639/2003 (BRASIL, 2004).

\section{Considerações Finais}


A Matemática, enquanto ciência, é ainda interpretada pelos adolescentes, na fase escolar, como um conhecimento distante da sua realidade, pois na escola é ensinado de forma direta ou não, que é algo que demandam muitas horas de estudo, discussão e pensamento. Vale lembrar, que ela é um saber que exige seriedade e sua a satisfação é adquirida quando se consegue solucionar um problema que levou certo tempo para ser resolvido.

A Matemática é um objeto de resistência tal qual a cultura Negra, conforme exposto no colóquio. Infelizmente, poucos estudantes conseguem construir saberes e relacioná-las com essa ciência, devido a "cultura do medo" ou do "desinteresse", ou seja, desde a infância, a criança é desmotivada a interagir e relacionar suas brincadeira e momentos de prazer com os números e suas aplicações. Separa-se a beleza da Matemática do cotidiano do ser humano.

Neste relato, mesmo de forma simples e despojada, em modelo remoto de ensino, que descreve a atuação de um simples professor da educação básica apresentando a relação entre a disciplina que leciona em sala com a história do povo preto em nosso país, é possível por meio desta proposta, identificar a grande contribuição afro-indígena para a Matemática através dos jogos Yoté e Mancala. Pois, reconhecemos no desenvolver deste trabalho, a simplicidade, a significação e transmissão de saberes de forma simples e despojada.

Para se aprender matemática, basta apenas se permitir aprender. Em tempos coloniais em que a escravidão era a principal fonte de economia, onde os povos africanos passavam horas em trabalho pesado, reconhecemos ao jogar o Mancala, a capacidade de representação e raciocínio lógico, a alegria não só em ser o vencedor, mas operar suas pedras até cada cala, e o processo de ensino aprendizagem e significação, onde vemos os mais velhos ensinarem que até em covas no chão são possíveis a realização do jogo.

É indispensável a abertura dos jovens estudantes ao conhecimento das gerações passadas, ou seja, das histórias dos avós, das comidas da infância, dos jogos de infância, pois é uma forma de contribuir para que o que seja positivo, não se perca com o decorrer dos tempos. E que consequentemente, são repletos de ancestralidade e significação.

Reconhecemos, através do estudo da ancestralidade desses jogos afro-indígena apresentados, a grande contribuição do povo preto na Matemática e pretendemos 
investigar mais ainda sua atuação não só no cálculo das operações e raciocínio lógico, mas nas demais subáreas como a geometria, cálculo mental entre outros.

\section{Referências}

\section{ANAIS DO ARTEFATOS DA CULTURA NEGRA, EDUCAÇÃO}

AFROPENSADA: repensar o currículo e construir alternativas de combate ao racismo, de 31 de agosto à 04 de setembro de 2015, Crato [recurso digital] / organizadores: Cicera Nunes; Karla Roberta Brandão de Oliveira; Ana Paula Santos. - Crato-CE: Universidade Regional do Cariri, 2015. 811p.

ANDRADE, J. LEAL, Y. MONTEIRO, A. ANDRÉ, R. MACLYNE, D. TELES, R. GITIRANA,V. Projeto Rede: Jogos na Educação Matemática, UFPE. Mankala Colhe Três. Disponível em:

<http://lematec.net/projetorede/uploads/Textos/Mancala\%20colhe\%20Tr\%C3\%AAs\%2 0-\%20regras\%20e\%20hist\%C3\%B3rico.pdf>. Acesso em 20 dez. 2011.

ASSOCIAÇÃO BRASILEIRA DE NORMAS TÉCNICAS. NBR 6022: Informação e Documentação - Artigo em publicação periódica científica impressa - Apresentação. Rio de Janeiro, 2003. 5 p.

BARRETO, Glaúcia Bonfim Barbosa. O Ensino de Matemática através de Jogos Educativos Africanos: Um Estudo de caso em uma turma de Educação de Jovens e Adultos (EJA) de uma Escola Municipal de Aracaju. Orientador: Ana Maria Freitas Teixeira. - São Cristóvão, 2016.134 f.: il. Dissertação (Mestrado em Ensino e Ciências Naturais e Matemática) - Universidade Federal de Sergipe, 2016.

BRANDÃO, Ana Paula. Modos de fazer: caderno de atividades, saberes e fazeres / [organização Ana Paula Brandão]. Rio de Janeiro: Fundação Roberto Marinho, 2010.il. (A cor da cultura; V. 4)

BRASIL. Ministério da Educação (MEC). Parâmetros curriculares nacionais para o ensino médio. Brasília: MEC, 2002, p. 87.

BRASIL. Lei ${ }^{\circ} 10.639$, de 9 de janeiro de 2003. Altera a Lei no 9.394, de 20 de dezembro de 1996, que estabelece as diretrizes e bases da educação nacional, para incluir no currículo oficial da Rede de Ensino a obrigatoriedade da temática "História e Cultura Afro-Brasileira", e dá outras providências. Diário Oficial [da] República Federativa do Brasil. Brasília, DF, 9 jan. 2003. Disponível em: < http://www.planalto.gov.br/ccivil_03/leis/2003/110.639.htm>. Acessado em 20 de set. 2020.

Presidência da República. Lei no 10.639 de 9 de janeiro de 2003. Brasília: Diário Oficial da União, 10 de janeiro de 2003. 
BRASIL. Ministério da Educação (MEC). Diretrizes curriculares nacionais para a educação das relações étnico raciais e para o ensino de história e cultura afro-brasileira e africana. Brasília: MEC, 2004, p. 16.

. Lei no 11.645 de 10 de março de 2008. Brasília: Diário Oficial da União, 11 de março de 2008.

Copyright (C) Cyningstan 2021: Cyningstan. 2021. Disponível em: $<$ http://www.cyningstan.com/game/342/yot> Acessado em 11 de fevereiro de 2021.

D’AMBROSIO, Ubiratan. Etnomatemática: um programa a educação matemática. Revista da Sociedade Brasileira de Educação Matemática, v. 1, n. 1, p. 5-11, 1993.

D'AMBRÓSIO, Ubiratan. Sociedade, cultura, matemática e seu ensino. Revista Educação e Pesquisa. São Paulo, v. 31, p. 99-120, 2005.

DONOVAN,Tristan: Ludopedia,2021. Disponível em:< https://www.ludopedia.com.br/topico/17786/semeando-os-pontos-a-historia-damancala?id_post=145406 > Acessado em 11 de fevereiro de 2021.

FRANÇA, Junia Lessa; VASCONCELLOS, Ana Cristina de. Manual para normalização de publicações técnico-científicas. 7. ed. Belo Horizonte: Ed. UFMG, 2004.

FRANÇA, Rodrigo. O pequeno príncipe preto. $1^{\text {a }}$ ed. Rio de Janeiro: Nova Fronteira,2020.

FREYRE, Gilberto. Casa-Grande \& Senzala. 43 ed. Rio de Janeiro: Record, 2001.

FOGAHOLY, Cláudia. SILVA, Júlio Cesar da. FERNANDES, Pedro Borges. VAZ, Paulo Vieira dos Santos. SILVA, Moisés Teixeira da. FERREIRAS, Matheus Garcia de Alvarenga. Jogos de Mancala nas aulas de Educação Física: possibilidades de um projeto de extensão. Revista do Departamento de Educação Física. Colégio Dom Pedro II. Temas em Educação Física Escolar, Rio de Janeiro, v. 4, n.2, ago./dez. 2016, p. $159-170$.

FUTURA TEC: A COR DA CULTURA. 2021. Disponível em:< http://www.acordacultura.org.br/artigos/29082013/a-influencia-africana-noprocessodeformacaodaculturaafrobrasileira\#: :text=Essas \%20trocas\%20culturais $\% 20 \mathrm{oc}$ orridas\%20por, \%2C \%20religi\%C3\%A3o\%2C\%20m\%C3\%BAsica\%20e \%201\%C3\%A Dngua.> Acessado em 11 de fevereiro de 2021.

GIL, A. C. Como elaborar projeto de pesquisa. 4. ed. São Paulo: Atlas, 2002. GONZAGA, Rhaysa,T .SANTANDER,A., Malu .REGIANI,M. Anelise. A cultura Afro-brasileira no Ensino de Química. Revista Nova Escola. São Paulo - SP- Brasil, Vol 41, nº1, p 25-32, Fevereiro de 2019.

IBGE. Normas de apresentação tabular. 3. ed. Rio de Janeiro, 1993. 
KURIANSK, Katia i: Canal Vem Jogar. 2021. Disponível em:< https://www.youtube.com/watch?v=I8xROnVNCjY>Acessado em 11 de fevereiro de 2021.

LAKATOS, Eva Maria.; MARCONI, Maria Andrade. Fundamentos da metodologia científica. 5. ed. São Paulo: Atlas, 2003

LE COADIC, Yves-François. Ciência da Informação. 2. ed. Brasília: Briquet de Lemos, 2004.

LIMA, Heloise Pires. A Semente que veio da África. São Paulo, Salamandra, 2005.

MACEDO, Lino de. Aprender com os Jogos e Situação Problema. Rio de Janeiro: Artmed, 2000.

MINAYO, M. C. de L. (Org.) Pesquisa social: teoria, método e criatividade. 19 ed. Petrópolis: Vozes, 2001.

MORAES, Marcelo José Derzi. A filosofia Ubuntu e o quilombo: a ancestralidade como questão filosófica. Revista África e Africanidades - Ano XII - n. 32, nov. 2019 ISSN 1983-2354

MOTA, Carla Gleici da Silva, et al. Cultura Africana nas aulas de Matemática: Combatendo o racismo e a aprendizagem de conceitos matemáticos. Revista de Educação da Universidade Federal do Vale do São Francisco (REVASF). Petrolina - PE - Brasil, Vol 10, n.23, p.390-4413, dezembro, 2020.ISSN:2177-8183. PeriódicosUNIVASF.

RIBEIRO, P. S. Cultura brasileira: da diversidade à desigualdade. Brasil Escola. Disponível em http://brasilescola.uol.com.br/sociologia/cultura-brasileira-diversidadedesigualdade.htm, acessada em janeiro 2019.

SEM AUTOR: Como se joga o Yoté?. BODOGEMU,2021.Disponível em: $<$ https://bodogemu.com/pt/games/yote>Acessado em 12 de fevereiro de 2021.

SEM AUTOR: Educa Criança.2021. Disponível em: <https://educacrianca.com.br/mancala > Acessado em 11 de fevereiro de 2021.

SEM AUTOR: Ludomania.2021. Disponível em:< http://www.ludomania.com.br/Tradicionais/mancala.html> Acessado em $11 \mathrm{de}$ fevereiro de 2021.

SOSIO, Andrea. Even more mancalas. Galeria de andrea.sosio. Flickr: andrea. sosio. Disponível em:<http://www.flickr.com/photos/andsos/galleries/72157626346391900/>. Acesso em: 26 fev. 2013. 